\title{
CONSTANT BEAMWIDTH GENERALISED SIDELOBE CANCELLER
}

\author{
Choo Leng Koh and Stephan Weiss \\ Communications Research Group, School of Electronics \& Computer Science, \\ University of Southampton, \\ Southampton, SO17 1BJ , United Kingdom \\ clk02r@ecs.soton.ac.uk, sw1@ecs.soton.ac.uk
}

\begin{abstract}
In this paper, we proposed a constant beamwidth discrete Fourier transform (DFT) beamformer based on the generalised sidelobe canceller (GSC). Broadband signals are decomposed into frequency bins which are grouped into octaves and tapered individually. The resulting beampattern possesses constant beamwidth across the entire operating spectrum, thus ensuring uniform spatial resolution. Further incorporation of the GSC allows adaptive nulling of interference to coincide with uniform resolution, enhancing the beamformer's performance. However, modification to the constraint equation of the standard GSC is required to account for the frequency-dependent weighting of sensors.
\end{abstract}

\section{INTRODUCTION}

Broadband beamforming using sensor arrays is an effective method for suppressing interference whose angle of arrival is different from the desired signal. There are two main approaches for broadband beamforming, based on either timedomain processing or frequency-domain processing. However, the frequency-domain approach is often preferred as it offers computational efficiency [1].

Spatial resolution of a beamformer is reciprocally proportional to both the aperture of the sensor array collecting the data and the frequency of the impinging waveform [2]. The beampattern shown in Fig. 2 illustrates the beamformer's gain response after convergence as a function of frequency and direction of arrival (DOA) for a uniformly spaced sensor array. Poor resolution can clearly be observed at lower frequencies indicated by the increase in beamwidth. Several methods have been suggested to reduce this spatial variation such as limiting the beamformer's operation to an octave frequency interval [3]. Other techniques consider the use of non-uniformly spaced arrays which may be obtained by judiciously thinning a uniformly spaced sensor arrangement $[4,5]$, the implementation of harmonic nesting [6] as well as the use of focusing matrices that transform data in each bin to a reference frequency bin [7], for the purpose of attaining frequency invariant property.

By contrast, the idea of this paper is to introduce a broadband beamforming structure that can be utilised over a frequency range larger than an octave, by suitably subdividing the array signals into different frequency bands that span no more than an octave. For each octave, sensor signals are extracted from different apertures of a non-uniformly spaced array. Thereby, higher frequency bands will be fed

The authors would like to thank Prof. Ian K. Proudler and Prof. John G. McWhirter of the Advanced Signal Processing Group, QinetiQ Ltd., Malvern, UK, for their support and technical input.

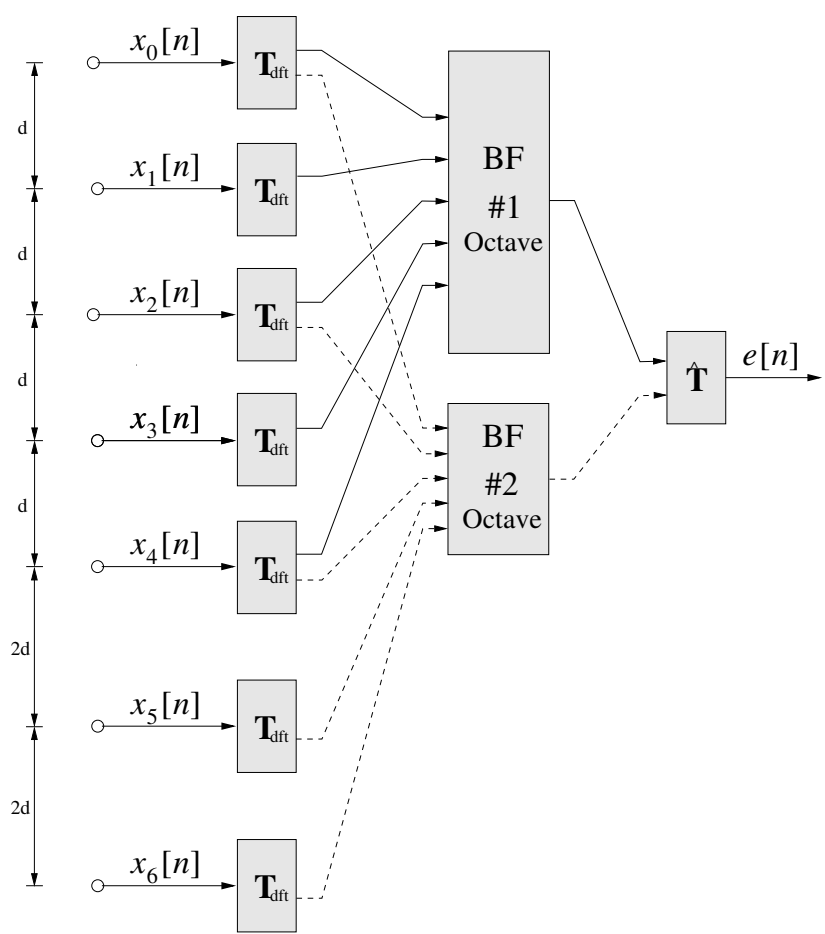

Figure 1: Scaled aperture beamformer.

from closely spaced sensors of small aperture, while low frequency bands operate on a wider spaced array of large aperture. A scaled aperture structure which decomposed a broadband signal into two octaves is depicted in Fig. 1, with beamformer \#1 processing signal of the higher octave and \#2 processes signal at the lower band. Each beamformer utilised $M=5$ sensor elements with $d$, the distance between adjacent sensors. This structure constitutes a tapered beamwidth for each octave, as illustrated by Fig. 3. To further enhance the uniformity of spatial resolution, signals from individual frequency bins are assigned different weight factors in accordance with the sensors positions. These weights emulate a specific beampattern and ensure constant beamwidth is maintained. Independent DFT-based GSC beamformers then process each frequency bin separately in an attempt to null out interfering signals while preserving the signal of interest.

The structure of the paper is as follows. In Sec. 2, a brief review of the time-domain and the DFT-based GSC beamformer are given along with the formulation of the constraint equation. Sec. 3 discusses the computation of sensor weights such that the beampattern can be suitably tapered to achieve 
$|\mathrm{A}(\Omega, \vartheta)| /[\mathrm{dB}]$

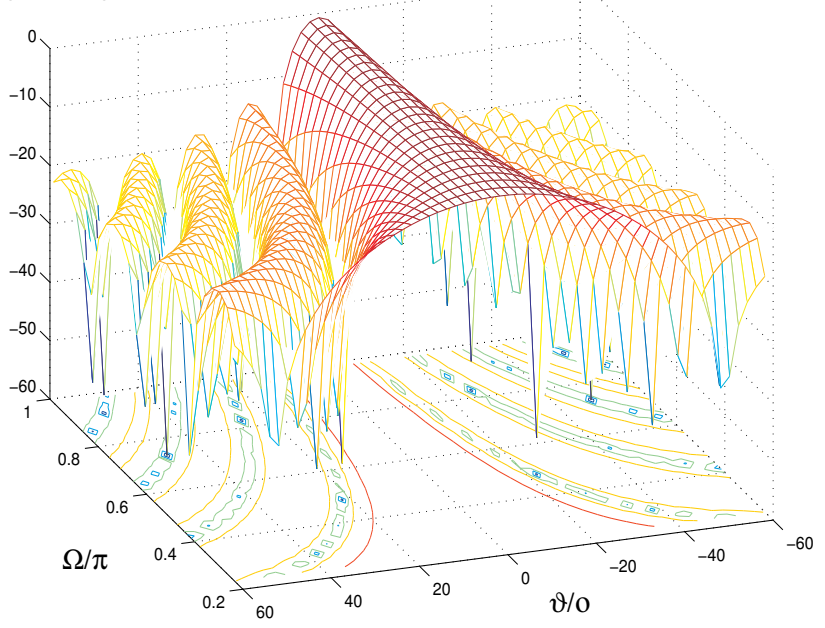

Figure 2: Directivity pattern of beamformer with fixed aperture.

$|\mathrm{A}(\Omega, \vartheta)| /[\mathrm{dB}]$

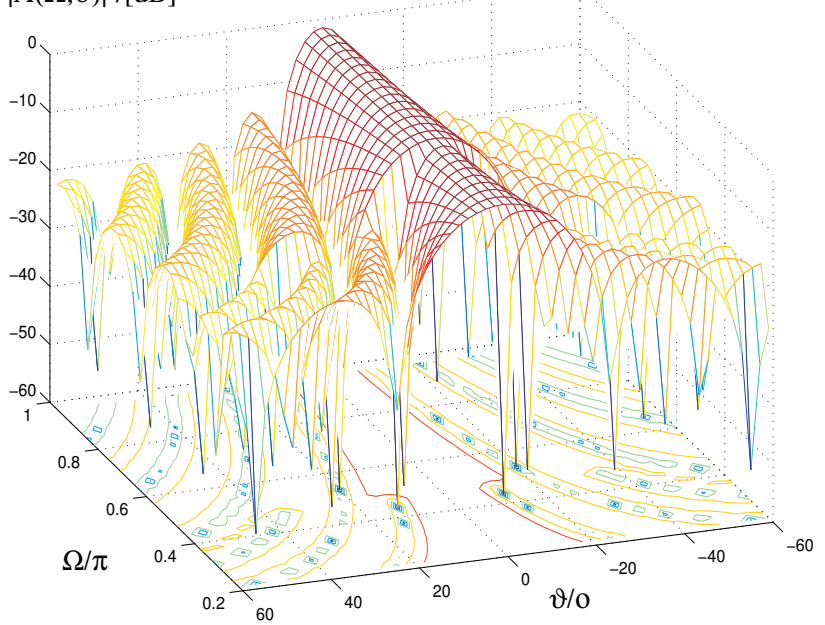

Figure 3: Directivity pattern of beamformer with scaled aperture.

constant resolution. The proposed constant beamwidth GSC structure, which incorporates the mechanism of Sec. 3 into a DFT-based GSC beamformer is detailed in Sec. 4. The associated simulation results achieved by the proposed system are compared with standard beamformers having fixed aperture in Sec. 5, while conclusion are drawn in Sec. 6.

\section{GENERALISED SIDELOBE CANCELLER}

The GSC structure evolves from a linearly constrained minimum variance (LCMV) beamformer, which performs a minimisation of the output power with respect to specific spatial and spectral constraints [8]. A time domain beamformer's output $e[n]$ can be written as

$$
e[n]=\mathbf{w}^{H} \mathbf{x}[n]
$$

where $\mathbf{x}[n]$ contains all the sensors' input samples in the delay line of filters $\mathbf{w}_{m}[n]$. The coefficients of these filters are collected in a single vector $\mathrm{w}$ formulated as

$$
\begin{aligned}
\mathbf{w} & =\left[\begin{array}{llll}
\mathbf{w}_{0}^{T} & \mathbf{w}_{1}^{T} & \cdots & \mathbf{w}_{M-1}^{T}
\end{array}\right]^{T}, \\
\mathbf{w}_{m} & =\left[\begin{array}{llll}
w_{0} & w_{1} & \cdots & w_{L-1}
\end{array}\right]^{T} .
\end{aligned}
$$

A similar definition applies for $\mathbf{x}[n]$, where $M$ and $L$ are the number of sensors and the filter length respectively.

The LCMV-based optimisation problem for the array weights can be represented by

$$
\mathbf{w}_{\text {opt }}=\arg \min _{\mathbf{w}} \mathbf{w}^{H} \mathbf{R}_{x x} \mathbf{w} \quad \text { subject to } \quad \mathbf{C}^{H} \mathbf{w}_{\text {opt }}=\mathbf{f},
$$

where matrix $\mathbf{C}$ is the $M L \times L$ constraint matrix, and $\mathbf{f}$ is the $L \times 1$ constraining vector and $\mathbf{R}_{x x}$ is the $M L \times M L$ autocorrelation matrix of the input signal vector $\mathbf{x}[n]$. This beamformer presents a constrained optimisation problem which may be solved by the Frost algorithm [9]. In contrast, the GSC allows unconstrained optimisation by appropriately projecting the input signal $\mathbf{x}[n]$ away from the constrain subspace by means of the blocking matrix $\mathbf{C}_{a}$ and a quiescent vector $\mathbf{w}_{q}$. Thus, allowing the use of standard optimisation algorithm such as the least mean square (LMS)[10].

The design of the constraint plays a significant role in the functionality of the GSC beamformer. In order to correctly handle the constraint design for the constant beamwidth beamformer later, a brief consideration of the fullband case is given. Assuming that the signal of interest (SOI) impinging onto the array from broadside is to be preserved. The constraint equation $\mathbf{C}^{H}$ is defined using $M$ identity matrices $\mathbf{I}_{L} \in \mathbb{R}^{L \times L}$ :

$$
\mathbf{C}^{H}=\underbrace{\left[\begin{array}{llll}
\mathbf{I}_{L} & \mathbf{I}_{L} & \cdots & \mathbf{I}_{L}
\end{array}\right]}_{M} .
$$

The constraining vector $\mathbf{f}$ which determines the desired impulse response of the array in the look direction is given by

$$
\mathbf{f}=\left[f_{0} f_{1} \cdots f_{L-1}\right] .
$$

For a reception indiscriminate of frequency, $\mathbf{f}$ could be a centred impulse.

Constraints for the DFT-based beamformer differ from the time-domain and are represented by,

$$
\mathbf{C}=\left[\begin{array}{cccc}
\mathbf{c}_{0} & \mathbf{0}_{M, 1} & \ldots & \mathbf{0}_{M, 1} \\
\mathbf{0}_{M, 1} & \mathbf{c}_{1} & \ldots & \mathbf{0}_{M, 1} \\
\vdots & \vdots & \ddots & \vdots \\
\mathbf{0}_{M, 1} & \mathbf{0}_{M, 1} & \ldots & \mathbf{c}_{L-1}
\end{array}\right]
$$

which is arranged according to frequency bins rather than sensors. If signal of interest (SOI) impinges from broadside, vector $\mathbf{c} \in \mathbb{C}^{M \times 1}$ is identical for all bins, a single c can be employed to compute the blocking matrix $\mathbf{C}_{a} \in \mathbb{C}^{M \times M-r}$ via singular value decomposition (SVD) [11], where $r$ is the number of linearly independent constraints. The function of this matrix is to block the frequency of interest for each bin. The quiescent vector for individual bins can be formulated as

$$
\mathbf{w}_{q, l}=\mathbf{c}\left(\mathbf{c}^{H} \mathbf{c}\right)^{-1} f_{l}^{\mathrm{dft}}
$$

where $f_{l}^{\mathrm{dft}}$ is the $l^{\text {th }}$ frequency component attained from the DFT of (5). These quiescent vectors are arranged in a matrix

$$
\mathbf{W}_{q}=\left[\mathbf{w}_{q, 0} \mathbf{w}_{q, 1} \cdots \mathbf{w}_{q, L-1}\right] \quad \in \mathbb{C}^{M \times L} .
$$




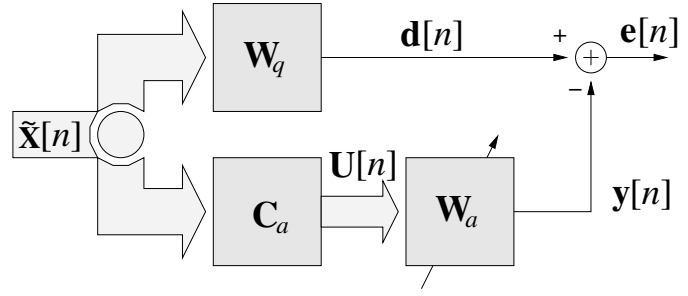

Figure 4: DFT-based GSC structure.

Note that this quiescent matrix now takes all frequency bins into consideration.

Fig. 4 portrays a DFT-based GSC structure where $\tilde{\mathbf{X}}[n] \in$ $\mathbb{C}^{M \times L}$ contains the frequency representation of the signal from $M$ sensors after Fourier transformation. The output of $\mathbf{W}_{q}$ is given by

$$
\mathbf{d}[n]=\operatorname{diag}\left(\mathbf{W}_{q}^{H} \tilde{\mathbf{X}}[n]\right),
$$

while the blocking matrix output is written as,

$$
\mathbf{U}[n]=\mathbf{C}_{a}^{H} \tilde{\mathbf{X}}[n] .
$$

The unconstrained adaptive coefficients in $\mathbf{W}_{a} \in \mathbb{C}^{M-r \times L}$ may be adapted using the LMS algorithm, resulting in an output vector $\mathbf{e}[n]$. A time domain output representation is attained by the summation of $\mathbf{e}[n]$ for the sliding window method while block processing requires an inverse DFT (IDFT) operation.

Note that this implementation assumes independent frequency bin processing. Thus, if the algorithm is excited by signal components off frequency bins, a degradation of performance is encountered [12]. A remedy to this problem is the use of the overlap-save technique [13], which is not consider here.

\section{SPATIAL TAPERING}

The solution to ensure constant beamwidth across a wide spectrum combines harmonic nesting with spatial tapering [6]. The harmonic nesting methodology starts by reducing a broadband beamforming problem into a set of octave based problems, with each octave having a different aperture. This results in a directivity pattern shown in Fig. 3, where the frequency variations within the octaves can be further tuned by frequency-dependent element weighting to achieve constant resolution. By assigning frequency independent weights to sensors, a beampattern that is constant across an octave can be achieved. We first review the design of a frequency dependent taper in order to obtain a frequency invariant beampattern within an octave. The choice of array pattern is the "periodic sinc" function, given by

$$
R[\vartheta]=\frac{1}{M} \frac{\sin \left(\frac{\pi M d}{\lambda_{1}} \vartheta\right)}{\sin \left(\frac{\pi d}{\lambda_{1}} \vartheta\right)},
$$

where $\lambda_{1}$ represents the wavelength of the lowest frequency component within an octave and $d$ is the distance between adjacent sensors. To avoid spatial aliasing $d$ must be kept at least half the wavelength of the highest frequency $\lambda_{\mathrm{u}}$ of the octave. Since the relationship between the longest and

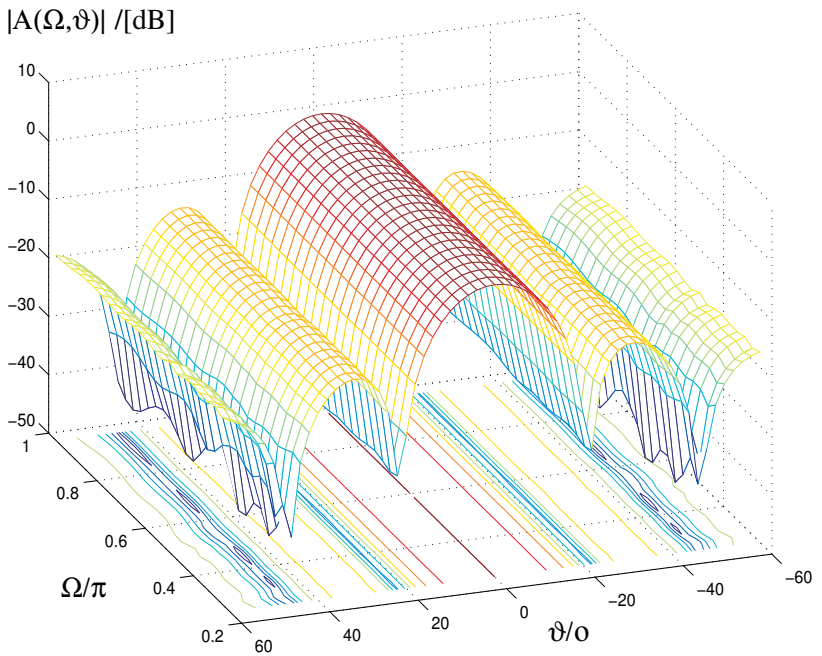

Figure 5: Directivity pattern of beamformer with constant beamwidth.

shortest wavelengths within an octave is $\lambda_{1}=2 \lambda_{\mathrm{u}}$, (11) can be simplified to

$$
R[\vartheta]=\frac{1}{M} \frac{\sin \left(\frac{\pi M}{4} \vartheta\right)}{\sin \left(\frac{\pi}{4} \vartheta\right)}
$$

To achieve constant beamwidth, the array pattern of (12) must be sampled at the interval of

$$
\vartheta_{m}=\frac{m}{M d \cdot \tilde{f}_{l}}, \quad-\frac{M-1}{2} \leq m \leq \frac{M-1}{2}, \quad 0 \leq l \leq L-1,
$$

for each frequency bin $\tilde{f}_{l}$. An IDFT is then performed on the sampled points to obtain weights of sensors for individual bins. These weighting when applied to the sensors, tapered the beampattern, ensuring constant beamwidth is maintained across the octave, as depicted in Fig. 5.

The spatial tapering technique allows uniform spatial resolution, but its inability to null out interference results in poor reception of the desired signal. Thus, the signal to interference ratio (SIR) may be unacceptable for certain cases. To mitigate this problem, our approach incorporates spatial tapering into a DFT-based GSC beamformer, as will be demonstrated in Sec. 4.

\section{CONSTANT BEAMWIDTH GSC}

For the proposed constant beamwidth GSC, consider the case where a broadband signal is decomposed into 32 frequency bins by means of a DFT. The frequency bins are subsequently grouped into 4 bands each having 8 frequency components. From the 4 bands, 2 octaves are formed, where the first octave contains band \#1 while the second has bands \#2 and \#3. Note that the lowest band \#0 containing a non-steerable DC component is neglected. The aperture size of the array is doubled when stepping from one octave to another. Due to independent frequency bin assumption assumed earlier, each octave is treated independently. Assuming that $M=11$ sensors are used, the aperture of the two octaves would be $10 \mathrm{~d}$ and $20 d$ respectively, where $d$ represents the distance between adjacent sensors. 
Within an octave, spatial tapering is performed by assigning different sets of weights to the sensors in accordance to their respective bins. These weights are calculated as described in Sec. 3. The resulting structure is a frequency invariant beamformer having uniform spatial resolution across the operating spectrum. For this GSC beamformer to effectively nulls out interferers, modifications to the constraint equation of the standard GSC is required to account for the frequency-dependent weighting of sensors. Since each sensor element in the array is weighted differently at each frequency bin, the constraining vector $\mathbf{c}$ which previously assumes uniform sensor weighting is no longer valid. Instead, different constraining vectors are required for individual bins of the DFT-based GSC beamformer. This new constraint takes the form of

$$
\hat{\mathbf{c}}_{l}=\operatorname{diag}\left(\mathbf{s}_{l} \mathbf{c}_{l}^{T}\right),
$$

where $\mathbf{s}_{l} \in \mathbb{R}^{M \times 1}$ contains the tapered weights assigned to the sensors at the $l^{\text {th }}$ frequency bin. The values of $\mathbf{s}_{l}$ correspond to the IDFT of the array pattern sample points in (13). Unlike the broadside constraints described in Sec. 2, a single blocking matrix is no longer sufficient and individual blocking matrices have to be computed using (14) to ensure that signals are blocked correctly. The new blocking matrix takes the form of

$$
\mathbf{C}_{a}=\left[\begin{array}{llll}
\mathbf{C}_{a, 0} & \mathbf{C}_{a, 1} \cdots \mathbf{C}_{a, L-1}
\end{array}\right] \quad \in \mathbb{C}^{M \times L(M-r)},
$$

with each $\mathbf{C}_{a, l}$ applied to $\tilde{\mathbf{X}}_{l}[n]$. The same principle applies to the quiescent matrix $\mathbf{W}_{q}$, having a dimension of $M \times L$. Standard adaptive algorithms can be applied to the adaptive filter $\mathbf{W}_{a} \in \mathbb{C}^{M-r}$, for interference cancellation. If jamming is successful, the proposed beamformer will fulfill frequency invariancy coupled with correct cancellation of interference.

\section{SIMULATIONS}

In the following, we simulate the proposed constant beamwidth $(\mathrm{CB})$ structure for both sliding window and block processing methods. Results are compared with beamformers using similar DFT processing techniques but employ uniformly weighted equispace sensor array. Additionally, a fixed aperture fullband structure is used as a benchmark. Both the sliding window and block processing methods are DFT-based, with the former processing data at every time instance, while updating is done after a predefined block size for the latter. Thus, the block processing technique exhibits a computational advantage over the sliding window method since it can be fully decimated by the block size, which is normally the filter length $L$. For the constant beamwidth GSC, a total of $M_{\mathrm{tot}}=16$ non-uniformly spaced sensors are used, with each octave having $M=11$ sensors. Fixed structure extract signals from $M=11$ uniformly spaced sensor elements, with an aperture size of $10 \mathrm{~d}$. All the simulated beamformers have filter length of $L=64$, spanning two octaves.

In our simulated scenario, a broadband source of interest impinges onto the array from broadside, $\vartheta=0^{\circ}$, corrupted by a set of narrowband interferers that coincides with the frequency bins. Their direction of arrival (DOA) is $\vartheta=-10^{\circ}$ at a signal to interferer ratio (SIR) of $-50 \mathrm{~dB}$. Additionally, spatially and temporally uncorrelated noise corresponding to a signal to noise ratio (SNR) of $-6 \mathrm{~dB}$ are

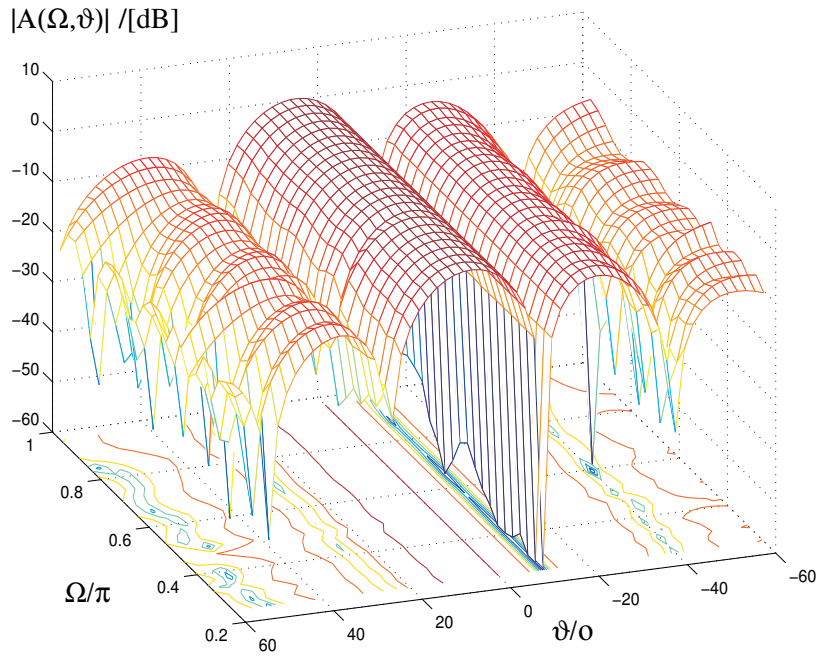

Figure 6: Directivity pattern of beamformer with constant beamwidth GSC.

inserted. Both sources are restricted to a normalised range of $\Omega=\{0.25 \pi ; \pi\}$. The adaptive algorithm used is the normalised LMS (NLMS).

The directivity pattern of the constant beamwidth GSC for the scenario outlined above is given in Fig. 6. Clearly a null is placed in the direction of the interference coming from a DOA of $-10^{\circ}$. Furthermore the OdB constraint towards broadside is fulfilled. Most importantly a constant beamwidth associated with uniform resolution across the simulated frequency range has been observed. Having uniform resolution does not always lead to a lower SNR output, rather it implies that the SNR across the frequency span is constant. This can be seen by comparing the beampattern of the scaled aperture depicted in Fig 3 with the constant beamwidth beamformer of Fig. 5. It reveals that constant beamwidth beamformer has the overall worse resolution. This is attributed to the limits placed on the aperture, which requires the used of the largest octave beamwidth associated with the scaled aperture. Referencing to a fixed aperture beamformer in Fig 2, scaled aperture improves the overall spatial resolution of a beamformer by an increase in the total number of sensors utilised.

The learning characteristic of the five different beamforming structures considered - namely the proposed constant beamwidth beamformers for both block and sliding window processing, the uniformly spaced linear array with DFT based block and sliding window technique and a fix aperture fullband beamformer are depicted in Fig 7. The step-size for all the beamformers is set to $\mu=0.1$, and results are averaged over an ensemble of 100 simulations. The mean square value of the residual error signal, which is the beamformer's output subtracted from the desired broadband signal, shows that DFT-based beamformers converge much faster than the time domain realisation. This improvement is due to the spectral whitening by having normalised step-size associated with the NLMS algorithm assigned independently to each frequency bin. In general sliding window techniques demonstrate a faster convergence speed compared to block processing. This is not surprising because adaptive coefficients are updated block by block while the updating pro- 


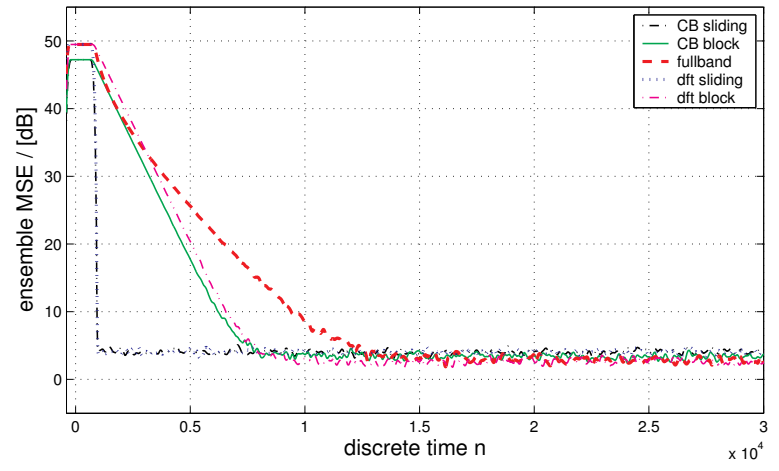

Figure 7: Learning curves of the beamformers.

cess for sliding window is carried out at every time instance. It can also be seen that the addition of the tapering process does not degrade the performance of the beamformer in the context of convergence speed and residue mean square error when compared against the corresponding DFT implementations.

\section{CONCLUSION}

The frequency invariant characteristic of broadband arrays, particulary the poor spatial resolution at low frequencies has motivated the introduction of a constant beamwidth GSC, which leads to constant resolution across a frequency range spanning more than an octave. This DFT-based frequency invariant beamformer also demonstrates the ability to adaptively null out interference. The block processing DFT-based beamformer offers higher computational efficiency, when compared to both time-domain and sliding window implementations, since it can be fully decimated. However, it exhibits a slower convergence speed compared to the sliding window method.

One problem associated with the DFT-based approach is the resulting poor performance when interferers do not sit on the frequency bins. Although not further discussed in this paper, the proposed constant beamwidth DFT-based beamformer suffers from a similar degradation. The overlapsave technique has demonstrated an ability to overcome this problem, and a new design based on a frequency invariant overlap-save GSC beamformer has been proposed [14].

\section{REFERENCES}

[1] L. C. Godara, "Application of the fast Fourier transform to broadband beamforming," Journal of the Acoustic Society of America, vol. 98, no. 1, pp. 230-240, July 1995.

[2] D. H. Johnson and D. E. Dudgeon, Array Signal Processing: Concepts and Techniques, Signal Processing Series. Prentice Hall, Englewood Cliffs, NJ, 1993.

[3] M. VanderWal and D. de Vries, "Design of Logarithmically Spaced Constant-directivity Transducer Arrays," Journal of the Audio Engineering Society, vol. 44, no. 6, pp. 497-507, 1996.

[4] G. Cardone, G. Cincotti, P. Gori, and M. Pappalardo, "Optimization of Wide-Band Linear Arrays," IEEE
Transactions on Ultrasonics, Ferroelectrics, and Frequency Control, vol. 48, no. 4, pp. 943-952, July 2001.

[5] R. M. Leahy and B. D. Jeffs, "On the Design of Maximally Sparse Beamforming Arrays," IEEE Transactions on Antennas and Propagation, vol. 39, no. 8, pp. 1178-1188, August 1991.

[6] T. Chou, "Frequency-independent beamformer with low response error," in Proc. IEEE International Conference on Acoustics, Speech, and Signal Processing, Detroit, Michigan, May 1995, vol. 5, pp. 2995-2998.

[7] D. B. Ward, R. A. Kennedy, and R.C. Williamson, "Theory and design of broadband sensor arrays with frequency invariant far-field beam patterns," Journal of the Acoustic Society of America, vol. 97, no. 2, pp. 1023-1034, Feburary 1995.

[8] L. J. Griffith and C. W. Jim, "An Alternative Approach to Linearly Constrained Adaptive Beamforming," IEEE Transactions on Antennas and Propagation, vol. 30, no. 1, pp. 27-34, January 1982.

[9] O. L. Frost, III, "An Algorithm for Linearly Constrained Adaptive Array Processing," Proceedings of the IEEE, vol. 60, no. 8, pp. 926-935, August 1972.

[10] K. M. Buckley, "Spatial/Spectral Filtering with Linearly Constrained Minimum Variance Beamformers," IEEE Transactions on Acoustics, Speech, and Signal Processing, vol. ASSP-35, no. 3, pp. 249-266, March 1987.

[11] K. M. Buckley and L. J. Griffith, "An Adaptive Generalized Sidelobe Canceller with Derivative Constraints," IEEE Transactions on Antennas and Propagation, vol. 34, no. 3, pp. 311-319, March 1986.

[12] S. Weiss and I. K. Proudler, "Comparing Efficient Broadband Beamforming Architectures and Their Performance Trade-Offs," in 14th International Conference on Digital Signal Porcessing, Santorini, Greece, July 2002.

[13] W. Liu, C. L. Koh, and S Weiss, "Constrained Adaptive Broadband Beamforming Algorithm in Frequency Domain," in Proc. IEEE Sensor Array and Multidimensional Signal Processing Workshop, Barcelona, Spain, July 2004, in press.

[14] C. L. Koh and S Weiss, "Overlap-Save Frequency Invariant Generalised Sidelobe Canceller," in Proc. International Symposium on Signal Processing and Its Applications, Sydney, Australia, August 2005, submitted. 
